\title{
ATRIAL LOW VOLTAGE AREAS: A COMPARISON BETWEEN ATRIAL FIBRILLATION AND SINUS RHYTHM
}

\author{
Ana Andres ${ }^{1}$, Carlos Roberto ${ }^{2}$, Francisco Javier Saiz ${ }^{3}$, Oscar Cano ${ }^{4}$, Laura \\ Martínez-Mateu ${ }^{3}$, Pau Alonso ${ }^{4}$, Assumpció Saurí ${ }^{5}$, Aurelio Quesada ${ }^{1}$, and Joaquin \\ Osca-Asensi ${ }^{6}$ \\ ${ }^{1}$ Universidad Catolica de Valencia San Vicente Martir Facultad de Medicina y Odontologia \\ ${ }^{2}$ Universitat Politècnica de València \\ ${ }^{3}$ Universitat Politecnica de Valencia \\ ${ }^{4}$ Hospital Politécnico y Universitario La Fe \\ ${ }^{5}$ Hospital Universitari i Politecnic La Fe \\ ${ }^{6}$ La Fe University and Polytechnic Hospital
}

November 2, 2020

\begin{abstract}
Aims Atrial fibrosis can promote atrial fibrillation (AF). Electroanatomic mapping (EAM) can provide information regarding local voltage abnormalities that may be used as a surrogate marker for fibrosis. Specific voltage cut-off values have been reproducibly shown to accurately identify fibrosis in the ventricles but they are not well defined in atrial tissue. Methods Unicenter prospective study. Consecutive patients with persistent AF referred for ablation were included. EAM was performed with Carto3 mapping system (Biosense Webster, Inc.). We recorded bipolar signals, first in AF and later in sinus rhythm (SR) after electrical cardioversion. Two thresholds delimited low-voltage areas (LVA): 0.5 and 0.3mV. We compared LVA extension between maps in SR and AF in each patient. Results 23 patients were included. Percentage of points with voltage lower than $0.5 \mathrm{mV}$ and $0.3 \mathrm{mV}$ was significantly higher in maps in AF compared to maps in SR: $38.2 \%$ of points $<0.5 \mathrm{mV}$ in $\mathrm{AF}$ vs. $22.9 \%$ of points $<0.5 \mathrm{mV}$ in $\mathrm{SR}(\mathrm{p}<0.001) ; 22.3 \%$ of points $<0.3 \mathrm{mV}$ in AF vs. $14 \%$ of points $<0.3 \mathrm{mV}$ in $\mathrm{SR}(\mathrm{p}<0.001)$. Areas with reduced voltage were significantly bigger in maps in $\mathrm{AF}(0.5 \mathrm{mV}$ threshold, mean area in $\mathrm{AF} 41.3 \mathrm{~cm} 2 \pm 42.5 \mathrm{~cm} 2 \mathrm{vs} 11.7 \mathrm{~cm} 2$ $\pm 17.9 \mathrm{~cm} 2$ in $\mathrm{SR}, \mathrm{p}<0.001 ; 0.3 \mathrm{mV}$ threshold, mean area in $\mathrm{AF} 15.6 \mathrm{~cm} 2 \pm 22.1 \mathrm{~cm} 2$ vs $6.2 \mathrm{~cm} 2 \pm 11.5 \mathrm{~cm} 2 \mathrm{in} \mathrm{SR}, \mathrm{p}<0.001)$. Conclusion Using the same voltage thresholds, LVA extension in AF is greater than in SR in patients with persistent AF. These findings provide arguments for defining a different atrial fibrosis threshold based on EAM rhythm.
\end{abstract}

\section{TITLE PAGE}

TITLE: ATRIAL LOW VOLTAGE AREAS: A COMPARISON BETWEEN ATRIAL FIBRILLATION AND SINUS RHYTHM

\section{AUTHORS:}

Ana Andrés ${ }^{1,2}$, Carlos Roberto ${ }^{3}$, Francisco Javier Saiz ${ }^{3}$, Óscar Cano ${ }^{2}$, Laura Martínez-Mateu ${ }^{3}$, Pau Alonso ${ }^{2}$, Assumpció Saurí ${ }^{2}$, Aurelio Quesada ${ }^{1}$, Joaquín Osca ${ }^{2}$.

1. Escuela de Doctorado. Universidad Católica de Valencia San Vicente Mártir.

2. Unidad de Arritmias. Hospital Universitario y Politécnico La Fe, Valencia.

3. Universitat Politècnica de València.

Funding: None 
Disclosures: None

Name of author to whom proofs should be sent: Ana Andrés Lahuerta. C/Maestro Palau 12, 7-14. CP 46008, Valencia, Spain. Telephone: +34690100553. E-mail:anaandreslahuerta@gmail.com

\section{ABSTRACT}

\section{Aims}

Atrial fibrosis can promote atrial fibrillation (AF). Electroanatomic mapping (EAM) can provide information regarding local voltage abnormalities that may be used as a surrogate marker for fibrosis. Specific voltage cut-off values have been reproducibly shown to accurately identify fibrosis in the ventricles but they are not well defined in atrial tissue.

\section{Methods}

Unicenter prospective study. Consecutive patients with persistent AF referred for ablation were included. EAM was performed with Carto3 mapping system (Biosense Webster, Inc.). We recorded bipolar signals, first in AF and later in sinus rhythm (SR) after electrical cardioversion. Two thresholds delimited low-voltage areas (LVA): 0.5 and $0.3 \mathrm{mV}$. We compared LVA extension between maps in SR and AF in each patient.

\section{Results}

23 patients were included. Percentage of points with voltage lower than $0.5 \mathrm{mV}$ and $0.3 \mathrm{mV}$ was significantly higher in maps in AF compared to maps in SR: $38.2 \%$ of points $<0.5 \mathrm{mV}$ in AF vs. $22.9 \%$ of points $<0.5 \mathrm{mV}$ in SR ( $\mathrm{p}<0.001) ; 22.3 \%$ of points $<0.3 \mathrm{mV}$ in $\mathrm{AF}$ vs. $14 \%$ of points $<0.3 \mathrm{mV}$ in $\mathrm{SR}(\mathrm{p}<0.001)$. Areas with reduced voltage were significantly bigger in maps in $\mathrm{AF}\left(0.5 \mathrm{mV}\right.$ threshold, mean area in $\mathrm{AF} 41.3 \mathrm{~cm}^{2} \pm$ $42.5 \mathrm{~cm}^{2}$ vs $11.7 \mathrm{~cm}^{2} \pm 17.9 \mathrm{~cm}^{2}$ in $\mathrm{SR}, \mathrm{p}<0.001 ; 0.3 \mathrm{mV}$ threshold, mean area in $\mathrm{AF} 15.6 \mathrm{~cm}^{2} \pm 22.1 \mathrm{~cm}^{2}$ vs $6.2 \mathrm{~cm}^{2} \pm 11.5 \mathrm{~cm}^{2}$ in SR, p <0.001).

\section{Conclusion}

Using the same voltage thresholds, LVA extension in AF is greater than in SR in patients with persistent AF. These findings provide arguments for defining a different atrial fibrosis threshold based on EAM rhythm.

\section{INTRODUCTION}

Atrial fibrillation (AF) is the most common cardiac tachyarrhythmia.. Its pathophysiology is complex ${ }^{1}$ and can be described with: 1) triggers for the initiation of the arrhythmia (pulmonary and non-pulmonary foci); 2) a fibrotic substrate for the maintenance of AF; and 3) various modulators acting by multiple potential mechanisms (hypertension, obesity, obstructive sleep apnea, inflammation, endurance sports...).

Atrial remodeling and fibrosis development are associated with a variety of electric disturbances, such as heterogeneities in atrial action potential duration, effective refractory period, and conduction velocity ${ }^{2}$. These phenomena can promote and sustain $\mathrm{AF}$.

Left atrial (LA) scarring can be detected by late enhancement MRI (MRI-DE) and can be correlated well with reduced electrogram amplitudes as recorded by endocardial voltage maps ${ }^{3}$. However, MRI to assess atrial fibrosis is not available in all centers due to its complex evaluation.

Electroanatomic mapping (EAM) can provide information regarding local voltage abnormalities that may be used as a surrogate marker for fibrosis. Specific voltage cut-off values have been reproducibly shown to accurately identify scar and/or fibrosis in the ventricles ${ }^{4,5}$. EAM voltage cut-off values to identify myocardial scars in the atrial tissue are not as well defined as in the ventricle. Voltage-guided AF substrate modification targeting low voltage areas (LVA) has been carried out in some studies to improve long-term AF ablation efficacy ${ }^{6-11}$. In most of them mapping has been performed using voltage cut-offs during sinus rhythm (SR), and in one study mapping was performed during $\mathrm{AF}^{11}$. However, a recent study has documented that the correlation between low-voltage and posterior LA MRI-DE is significantly improved when acquired during AF vs. sinus rhythm ${ }^{12}$. 
The aim of our study was to evaluate the LVA extent and location in patients with persistent AF undergoing pulmonary veins isolation, and compare the findings on maps obtained in SR and AF in each patient.

\section{METHODS}

This was a unicenter prospective study. Patient demographics, clinical characteristics, and medications were exported from patient records. All participants provided written informed consent for the ablation procedure and for the inclusion in medical research at the time of procedure.

\section{Study population}

All consecutive patients with persistent AF referred to our center for pulmonary vein isolation (PVI) were included (between September 2016 and June 2019). Exclusion criteria were redo ablations (to avoid bias owing to prior ablations, which could affect AF substrate) and patients who arrived in SR the procedure day.

\section{Electroanatomic Mapping and Ablation}

All procedures were performed under general anesthesia. Antiarrhythmic agents, if present, were not discontinued. Patients maintained anticoagulant treatment (acenocumarol with INR from 2 to 3.5, or direct oral anticoagulants, with last dose the night before the procedure).

After vascular access was obtained, a double transseptal puncture was performed and intravenous heparin was administered to maintain an activated clotting time of more than 300 seconds. Thereafter, two long sheaths (1 SL0 sheath and 1 Agilis sheath; St. Jude Medical, Inc., St. Paul, MN) were inserted into the left atrium. The following catheters were used: a decapolar catheter WEBSTER B CS Catheter (Biosense

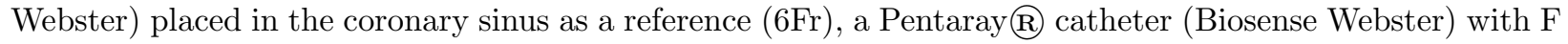
curve 2-6-2 $\mathrm{mm}$ spacing between electrodes (7Fr) as a high density mapping catheter (10 pairs of electrodes) and a SmartTouch SF with an F curve as an irrigated-tip contact force ablation catheter (7.5Fr).

Three-dimensional geometry of the left atrium and 4 pulmonary veins (PVs) was reconstructed with the use of Carto3 mapping system (Biosense Webster, Inc.). To ensure that mapping catheter is in contact with the tissue, CARTO system features the TPI or Tissue Proximity Indicator, which performs an impedance matrix. When it contacts with the cardiac wall, the catheter has less ion-charged blood, so impedance rises. To carry out the automatic acquisition of points, a series of filters were included. Those used for the acquisition of these maps are the following: Cicle Lenght Filtering (not in AF), Local Activation Time Stability (not in AF), Position Stability, Density and Respiration Gating.

We recorded multiple bipolar signals (filter setting: 30-300 Hz) from the Pentaray catheter, first in AF and later in SR after electrical cardioversion. Operators mapped carefully to ensure that the entire left atrium anatomy was represented in both the SR and the AF maps. All points within the pulmonary veins and LA appendage were excluded. After both electroanatomical maps were completed, ablation was performed as usual in our center: ipsilateral PV isolation in pairs, with entrance and exit block as the electrophysiological endpoint. In some cases, ablation could also include lines of ablation, at operator's discretion.

All procedures were performed by two expert operators.

\section{Fibrosis area measurement}

Two thresholds were established to delimit LVA: $0.5 \mathrm{mV}$ and $0.3 \mathrm{mV}$. We added the condition of presenting voltages higher than $0.1 \mathrm{mV}$ to avoid areas of dense fibrosis and possible false voltages due to contact problems.

To delimitate LVA extension, we used a tool present in CARTO called "Area Measurement". With this tool, low voltage extension is manually drawn according to the colour code given by the chosen thresholds. Since this measurement process is performed manually, there may be discrepancies between one measurement and another. In order to eliminate variability, two observers make two measurements of each patient. 


\section{Qualitative index of fibrosis distribution in $A F$ and $S R$}

In order to correlate location of LVA between AF and SR maps, a qualitative index was defined based on 4 projections (antero-posterior (AP), postero-anterior (PA), superior (SUP) and inferior (INF)). In every view, LVA in SR and AF were compared, assigning different values according to the degree of similarity. In this way, a value of 0 would correspond to discrepancies in the location of LVA between both maps; if LVA were located in the same zones, the assigned value would be 2 . For cases where there were no LVA in SR and therefore the comparison was not possible, the assigned value would be 1 . Finally, values obtained for each projection were added, obtaining a final value. If this value was equal to or greater than 5 (which means that at least one projection coincides and the other 3 are not evaluable), it was defined as correspondence between LVA in both maps. This process was performed for both thresholds, 0.5 and $0.3 \mathrm{mV}$.

\section{Percentage of fibrosis}

We calculated a surrogate of fibrosis studying the percentage of LVA with $0.5 \mathrm{mV}$ threshold. This percentage was calculated as the relationship between the area with voltage $<0.5 \mathrm{mV}$ and the total area. We analysed it in both maps (AF and SR). In a newly created qualitative variable to assess fibrosis, we assigned a value of 1 when the percentage of LVA was greater than $5 \%$.

Follow-up

Antiarrythmic medications were continued based on medical criteria. We considered a blanking period of 3 months after the ablation procedure during which the arrhythmia recurrences were not judged as ablation failure. All patients underwent ambulatory medical control visits after 6 and after 12 months of the ablation procedure. At each time, a 12-lead ECG and 24-hour holter ECG were recorded. If patients presented symptoms earlier, they underwent additional 12-lead ECG and 24 hour holter ECG. We considered arrhythmic recurrence at least one episode of sustained AF or atrial flutter ( $>30$ seconds) recorded on a surface 12-lead ECG or holter ECG.

\section{Statistical analysis}

IBM SPSS statistics package was used for statistical analysis. Numerical data were tested for normality using the Shapiro-Wilk test. Numerical variables were expressed as mean +- standard deviation. Categorical variables were expressed in percentages. Paired and unpaired t tests were used when appropriate. Categorical variables were compared between groups by using chi square test. The significance level was defined as $\mathrm{p}<$ 0.05. To evaluate interobserver concordance, the intraclass correlation coefficient was calculated.

\section{RESULTS}

A total of 23 patients were included. Patients' characteristics are shown in table 1.

All the studied variables had a normal distribution by the Shapiro-Wilk test.

\section{Procedure}

All patients were in AF before the procedure. Acute PVI was achieved in all patients. Two of them also had a history of common isthmus-dependent atrial flutter, so cavotricuspid isthmus ablation was also performed. Ablation lines were performed in three patients who presented atypical left atrial flutter during the procedure.

Median procedure time (from femoral venous puncture to removal of all catheters) was 169.3+-29 minutes and median fluoroscopy time was $5.6+-2.5$ minutes.

The only complication derived from the procedure was one case of cardiac tamponade (4.3\%) that was solved by pericardiocentesis.

Electroanatomic mapping and low voltage area study

Number of points 
Mean number of points for maps in AF was 3428+-1159 points, whereas it was 2319+-1143 points for maps in SR.

\section{Percentage of points}

Percentage of points with voltage lower than $0.5 \mathrm{mV}$ and with voltage lower than $0.3 \mathrm{mV}$ was significantly higher in maps in AF compared to maps in SR: $38.2 \%$ of points $<0.5 \mathrm{mV}$ in maps in AF vs. $22.9 \%$ of points $<0.5 \mathrm{mV}$ in maps in $\mathrm{SR}(\mathrm{p}<0.001) ; 22.3 \%$ of points $<0.3 \mathrm{mV}$ in maps in $\mathrm{AF}$ vs. $14 \%$ of points $<0.3 \mathrm{mV}$ in maps in SR $(\mathrm{p}<0.001)$.

\section{Mean voltage}

Global mean voltage of maps in AF was significantly lower than mean voltage of maps in SR: $0.62 \mathrm{mV}+-0.27$ $\mathrm{mV}$ vs. $1.62 \mathrm{mV}+-0.7 \mathrm{mV}(\mathrm{p}<0.001)$. When we selected areas with voltage lower than $0.5 \mathrm{mV}$, significant differences in mean voltage were not observed between both types of maps $(0.277 \mathrm{mV}+-0.02 \mathrm{mV}$ vs. 0.272 $\mathrm{mV}+-0.01 \mathrm{mV}, \mathrm{p} 0.27)$. No differences were found either in mean voltage in areas with voltage lower than $0.3 \mathrm{mV}(0.192 \mathrm{mV}+-0.008 \mathrm{mV}$ in maps in $\mathrm{AF}$ vs. $0.92 \mathrm{mV}+-0.007 \mathrm{mV}$ in maps in SR, p 0.448).

\section{Low voltage area measurement}

For $0.5 \mathrm{mV}$ threshold, all patients had LVA when the maps were performed in AF, whereas only $52.2 \%$ of patients had LVA when the maps were performed in SR. For $0.3 \mathrm{mV}$ threshold, $78.2 \%$ of patients had LVA in the AF maps, whereas only $43.5 \%$ of patients had LVA in the SR maps.

Although global area of maps in AF was smaller compared to maps in SR $\left(267.9 \mathrm{~cm}^{2}+-44.5 \mathrm{~cm}^{2}\right.$ vs.285.7 $\mathrm{cm}^{2}+-48.2 \mathrm{~cm}^{2}, \mathrm{p}<0.001$ ), areas with reduced voltages were significantly bigger in maps in AF. Specifically, for $0.5 \mathrm{mV}$ threshold, mean area of maps in AF was $41.3 \mathrm{~cm}^{2}+-42.5 \mathrm{~cm}^{2}$, while in maps in SR it was 11.7 $\mathrm{cm}^{2}+-17.9 \mathrm{~cm}^{2}, \mathrm{p}<0.001$. For $0.3 \mathrm{mV}$ threshold, mean area of maps in AF was $15.6 \mathrm{~cm}^{2}+-22.1 \mathrm{~cm}^{2}$, while it was $6.2 \mathrm{~cm}^{2}+-11.5 \mathrm{~cm}^{2}$ in maps in SR, $\mathrm{p}<0.001$.

Figures 1 and 2

\section{Qualitative index of assessment of fibrosis}

For the $0.5 \mathrm{mV}$ threshold, $91 \%$ of patients obtained a score equal to or greater than 5 points which, considering the description of our index, means that LVA in SR were present as LVA in maps in AF. The remaining $9 \%$ did not have LVA in SR maps, so location comparison could not be made. For the $0.3 \mathrm{mV}$ threshold, $52 \%$ of patients obtained a score equal to or greater than 5 points which, considering our index, means that LVA in SR were present in AF. In 39\% of patients, there were no LVA in maps in SR, so location comparison could not be made. Finally, in the remaining $9 \%$ of patients, the score was less than 4 points, so LVA in SR were not represented as LVA in AF.

Figures $3 A, 3 B, 4 A$ and $4 B$

\section{Interobserver correlation}

To assess interobserver correlation, the intraclass correlation coefficient was calculated based on the measurements carried out for each patient and each area by two independent observers. The results showed an adequate correlation with values greater than 0.8 in all cases:

Area measurement AF $<0.5 \mathrm{mV}$ : intraclass correlation coefficient $0.88 \mathrm{p}<0.001$

Area measurement AF $<0.3 \mathrm{mV}$ : intraclass correlation coefficient $0.92 \mathrm{p}<0.001$

Area measurement $\mathrm{SR}<0.5 \mathrm{mV}$ : intraclass correlation coefficient $0.89 \mathrm{p}<0.001$

Area measurement $\mathrm{SR}<0.3 \mathrm{mV}$ : intraclass correlation coefficient $0.86 \mathrm{p}<0.001$

Follow-up The mean follow-up time was $25+-12$ months. Ten patients ( $43.5 \%$ of all patients) presented arrhythmic recurrence at post-ablation follow-up. Among patients who had recurrence, 8 of them presented 
AF ( $80 \%$ of the patients with recurrence), whereas 2 of them presented atrial flutter ( $20 \%$ of the patients with recurrence)

Median arrhythmia free survival at follow-up was 25.3+-3.7 months.

For $0.5 \mathrm{mV}$ threshold, mean percentage of LVA in maps in SR was $4.2 \%$ whereas it was $15.6 \%$ in maps in AF. 2 tests were performed to assess a possible relationship between fibrosis and arrhythmic recurrences.

For the percentage of LVA in maps in AF the Pearson linear correlation test was $r=0.39$ with a value of $p$ closed to statistical significance $(\mathrm{p}=0.06)$. For maps in $\mathrm{SR}$, the result was $\mathrm{r}=0.189$, clearly not significant $(\mathrm{p}=0.388)$.

We studied the relationship of the percentage of LVA greater than $5 \%$ with arrhythmic recurrences. In AF, both variables showed almost significant association $(\mathrm{p}=0.06): 56.3 \%$ of patients that presented a percentage of LVA greater than $5 \%$ had arrhythmic recurrences whereas only $14.3 \%$ of patients with a percentage of LVA less than $5 \%$ had a recurrence. In SR, the result was not significant $(\mathrm{p}=0.382)$.

Figure 5

\section{DISCUSSION}

LVA studied by EAM of atrial bipolar endocardial signals have been recently established as a way to invasively define AF substrate. As an advantage, it does not require additional approaches or catheters and does not pose an additional risk to patients who, due to clinical criteria, undergo this therapeutic approach. Most authors consider these LVA as a surrogate of fibrosis ${ }^{13,14}$. Currently, we do not have a clear validation of a threshold that we have to consider as fibrosis. Some authors have adopted a statistical approach, considering the 5 th percentile of all mapping points as a threshold for abnormal tissue. For example, Kapa and collaborators ${ }^{15}$ proposed a threshold of $<0.2 \mathrm{mV}$ for atrial fibrosis in posterior wall and in the area between PV and left atrium, and $<0.45 \mathrm{mV}$ for the rest of the atrium, based on mapping of 20 patients with paroxysmal AF, 10 of them with previous PV isolation. Another study ${ }^{16}$ was carried out in patients with left accessory pathway ablation, some of them with additional AF. In patients without AF, $95 \%$ of electrograms voltage was greater than $0.38 \mathrm{mV}$, so they defined fibrosis voltage threshold in $0.4 \mathrm{mV}$. Other studies have been carried out in patients with supraventricular tachycardia undergoing left atrium mapping: Saghy ${ }^{17}$ established a cut-point between fibrotic and healthy tissue in $0.5 \mathrm{mV}$ in a study of 9 patients, while Yagashita ${ }^{18}$ proposed $1.17 \mathrm{mV}$ in a study of 6 patients. In the literature, the most widely used voltage value is $0.5 \mathrm{mV}$, but it may not be a threshold sensitive enough to identify areas with arrhythmogenic potential. Several studies ${ }^{6-11}$ have assessed the usefulness of an individualized ablation guided by LVA and most of them have used the $0.5 \mathrm{mV}$ threshold. In nearly all of them, LVA ablation has shown favourable results, what would support, although without great certainty, that this threshold level may be correct. However, these studies have important methodological differences, some of them in mapping that could influence LVA determination. These differences include mapping catheter electrodes spacing, electrode size, tissue contact, signal filtering, map's number of points and heart rhythm during mapping. In our study we used a multielectrode catheter with electrode size of $1 \mathrm{~mm}^{2}$, similar to that used in Yang ${ }^{19}$ and $\mathrm{Jadidi}^{20}$ studies, while the rest of studies used larger catheters. In healthy tissue, large catheters can cause a higher voltage record, whereas in areas with some fibrosis, a voltage summation of healthy and fibrotic voltages can result in lower amplitude signals ${ }^{21}$. Our mapping catheter, despite not having a direct contact force measurement, does have a tool that confirms proper contact before signal recording of a point. Adequate and consistent tissue contact is essential to avoid underestimation of endocardial signals voltage. Regarding the maps' number of points, the present work presents the advantage of high-density mapping. All maps presented more than 400 points, with a mean number of points in AF maps of 3428 and in SR map of 2319 points, much higher than average number of points from previous studies (100-200 points) with a maximum in Jadidi's study ${ }^{22}$ with 1024 points.

The main strength of our work lies in its objective: comparison of LVA according to maps' rhythm. In none of the exposed studies, mapping has been carried out in both rhythms in the same patient. Our results 
show that for a certain voltage threshold, LVA extension is greater in AF maps than in SR maps. Indeed, for the $0.5 \mathrm{mV}$ threshold, the mean area in AF maps was $41.3+-42.5 \mathrm{~cm}^{2}$ whereas in SR maps was 11.7 +- $17.9 \mathrm{~cm}^{2}(\mathrm{p}<0.001)$. For the $0.3 \mathrm{mV}$ threshold, it was $15.6+-22.1 \mathrm{~cm}^{2}$ in AF maps and $6.2+-11.5$ $\mathrm{cm}^{2}$ in SR maps $(\mathrm{p}<0.001)$. Another finding in the same vein was that the percentage of points below both thresholds was higher in AF maps than in SR maps. Likewise, the mean voltage of AF maps was significantly lower than in $\mathrm{SR}$ maps $(0.62 \mathrm{mV}+-0.27 \mathrm{mV}$ in $\mathrm{AF}$ vs. $1.62+-0.7 \mathrm{mV}$ in $\mathrm{SR}, \mathrm{p}<0.001)$. Since fibrosis is defined histologically and cannot be modified depending on heart rhythm, these data support that acquisition mapping rhythm implies a variation in the voltage recorded at each endocardial point. In fact, as early as 2003, Ndrepepal ${ }^{23}$ group assessed mean voltage in the left and the right atrium in SR and AF and observed that the voltage was significantly reduced when mapping was in AF. In addition, they observed that areas with the shortest AF length cycle had a greater voltage difference between AF and SR, suggesting a possible effect of rapid and disorganized depolarization on collected voltages. More recently, a Spanish study $^{24}$,carried out with a very accurate methodology, goes further and compares voltages of selected points in SR maps and in arrhythmia maps (in some cases AF and in others atrial flutter). They established that a value of $0.5 \mathrm{mV}$ in $\mathrm{SR}$ maps corresponds to $0.38 \mathrm{mV}$ in atrial flutter maps and to $0.31 \mathrm{mV}$ in AF maps. With these data and with those obtained in our work, we consider that it is necessary to establish different voltage thresholds depending on the rhythm in which mapping is performed. Furthermore, these thresholds should be generalized to scientific studies that evaluate LVA ablation impact. Also, parameters and tools for mapping should be standardized so they could be properly compared and generalized to clinical practice in case of favourable results.

Since LVA were less extensive in SR maps, we compared the SR LVA location to the AF LVA location. The qualitative scale we used did not allow comparison if there were not LVA areas in SR maps. We checked that in most of patients, LVA in SR were present in maps in AF. From our point of view, these data indicate that LVA in SR probably correspond to fibrosis, but it does not allow us to determine if the $0.5 \mathrm{mV}$ threshold supposes an overestimation of fibrosis zones in AF or an underestimation of these areas in SR.

In respect of post-ablation clinical evolution, we must emphasize that our data is only descriptive and exploratory for further investigations. Despite the fact that statistical significance is not reached in either of the two type of maps, if we consider LVA as a marker of fibrosis and therefore as a possible factor influencing post-ablation outcomes, we found, for the $0.5 \mathrm{mV}$ threshold, an almost significant relationship between recurrences and absolute LVA percentage and LVA percentage greater than $5 \%(\mathrm{p}=0.06)$. This does not happen at all with data in SR, whose results are far from significance. Although not clearly stated, it is possible that if patient sample had been more numerous, extension of LVA in AF would have been a factor clearly related to arrhythmic recurrences in post-ablation follow-up.

\section{LIMITATIONS}

It stands out that this is a study with few patients, although its sample number is similar to many previous publications that address atrial EAM. Areas in AF and SR were both measured manually, that may cause operator-dependent variations. However, intraclass correlation coefficients show adequate inter-operator agreement. Because of this, this factor influence seems to play a minor role. The study was not designed and does not have a sufficient sample size to assess evolutionary data, so obtained results are only exploratory.

\section{CONCLUSION}

Using the same voltage thresholds, LVA extension in AF is greater than in SR for patients with persistent $\mathrm{AF}$. Location of LVA in SR is present in AF in most of the patients. These findings provide arguments for defining a different atrial fibrosis threshold based on EAM rhythm.

\section{REFERENCES}

1. Kottkamp H, Schreiber D. The substrate in "earlypersistent" atrial fibrillation:Arrhythmia induced, riskfactor induced, or from a specific fibrotic atrial cardiomyopathy? J Am CollCardiol EP 2016;2:1402 . 
2. Li D, Melnyk P, Feng J, Wang Z, Petrecca K, Shrier A, Nattel S. Effectsof experimental heart failure on atrial cellular and ionic electrophysiology. Circulation. 2000;101:2631-2638.

3. Mahnkopf C, Badger TJ, Burgon NS, Daccarett M, Haslam TS, BadgerCT, McGann CJ, AkoumN, KholmovskiE, Macleod RS, Marrouche NF.Evaluation of the left atrial substrate in patients with lone atrial fibrillationusing delayed-enhanced MRI: implications for disease progression andresponse to catheter ablation. Heart Rhythm. 2010;7:1475-1481

4. GarciaFC, Dixit S, Lin D, TzouWS, Cooper JM, Verdino RJ, Callans DJ,Marchlinski FE: Endocardial unipolar voltagemapping to detect epicardialventricular tachycardia substrate in patients with nonischemic leftventricular cardiomyopathy. CircArrhythmElectrophysiol2011;4:49-55.

5. Hsia HH, Callans DJ, Marchlinski FE: Characterization of endocardialelectrophysiological substrate in patients with nonischemic cardiomyopathyand monomorphic ventricular tachycardia. Circulation2003;108:704-710.

6. Rolf S, Kircher S, Arya A, et al. Tailored atrialsubstrate modification based on low-voltage areasin catheter ablation of atrial fibrillation. CircArrhythmElectrophysiol2014;7:825-33.

7. Wang XH, Li Z, Mao JL, He B. A novel individualized substrate modification approach for thetreatment of long-standing persistent atrialfibrillation: preliminary results. Int J Cardiol 2014; 175:162-8.

8. Cutler MJ, Johnson J, Abozguia K, et al. Impactof voltage mapping to guide whether to performablation of the posterior wall in patients with persistent atrial fibrillation. $\mathrm{J}$ CardiovascElectrophysiol2016;27:13-21.

9. Jadidi AS, Lehrmann H, Keyl C, et al. Ablationof persistent atrial fibrillation targeting low voltage areas with selective activation characteristics. CircArrhythmElectrophysiol 2016;9.

10. Yamaguchi T, Tsuchiya T, Nakahara S, et al.Efficacy of left atrial voltage-based catheterablation of persistent atrial fibrillation.J CardiovascElectrophysiol2016;27:1055-63.

11. Blandino A, Bianchi F, Grossi S, et al. Left atrialsubstrate modification targeting low-voltageareas for catheter ablation of atrial fibrillation: a systematic review and meta-analysis. Pacing ClinElectrophysiol2017;40:199-212.

12. Norman Qureshi, Steven Kim, Chris Cantwell et al. Voltage during atrial fibrillation is superior to voltage during sinus rhythm in localizing areas of delayed enhancement on magnetic resonance imaging: An assessment of the posterior left atrium in patients with persistent atrial fibrillation. HeartRhythm, Vol -, No -, - 2019 (in press)

13. Kottkamp H. Human atrial fibrillation substrate: towards a specific fibrotic atrial cardiomyopathy. Eur Heart J. 2013;34(35):2731-2738. doi:10.1093/eurheartj/eht194

14. Callans DJ, Ren JF, Michele J, Marchlinski FE, Dillon SM. Electroanatomic left ventricular mapping in the porcine model of healed anterior myocardial infarction: Correlation with intracardiac echocardiography and pathological analysis. Circulation. 1999;100(16):1744-1750. doi:10.1161/01.CIR.100.16.1744

15. Kapa S, Desjardins B, Callans DJ, Marchlinski FE, Dixit S. Contact electroanatomic mapping derived voltage criteria for characterizing left atrial scar in patients undergoing ablation for atrial fibrillation. J Cardiovasc Electrophysiol. 2014;25(10):1044-1052. doi:10.1111/jce.12452

16. Lin Y, Yang B, Garcia FC, et al. Comparison of left atrial electrophysiologic abnormalities during sinus rhythm in patients with different type of atrial fibrillation. J Interv Card Electrophysiol. 2014;39(1):5767. doi:10.1007/s10840-013-9838-y

17. Saghy L, Callans DJ, Garcia F, et al. Is there a relationship between complex fractionated atrial electrograms recorded during atrial fibrillation and sinus rhythm fractionation? Hear Rhythm. 2012;9(2):181188. doi:10.1016/j.hrthm.2011.09.062

18. Yagishita A, Sparano D, Cakulev I, et al. Identification and electrophysiological characterization of early left atrial structural remodeling as a predictor for atrial fibrillation recurrence after pulmonary vein isolation. J Cardiovasc Electrophysiol. 2017;28(6):642-650. doi:10.1111/jce.13211

19. Yang G, Yang B, Wei Y, et al. Catheter Ablation of Nonparoxysmal Atrial Fibrillation Using Electrophysiologically Guided Substrate Modification during Sinus Rhythm after Pulmonary Vein Isolation.Circ Arrhythmia Electrophysiol. 2016;9(2). doi:10.1161/CIRCEP.115.003382

20. Jadidi AS, Lehrmann H, Keyl C, et al. Ablation of Persistent Atrial Fibrillation Targeting Low-Voltage 
Areas With Selective Activation Characteristics.[Erratum appears in Circ Arrhythm Electrophysiol. 2016 Jun;9(6). pii: e000015. doi: 10.1161/HAE.0000000000000015; PMID: 27255962]. Circ Arrhythmia Electrophysiol. 2016;9(3). doi:http://dx.doi.org/10.1161/CIRCEP.115.002962

21. Anter E, Tschabrunn CM, Josephson ME. High-Resolution Mapping of Scar-Related Atrial Arrhythmias Using Smaller Electrodes with Closer Interelectrode Spacing. Circ Arrhythmia Electrophysiol. 2015;8(3):537-545. doi:10.1161/CIRCEP.114.002737

22. Jadidi AS, Cochet H, Shah AJ, et al. Inverse relationship between fractionated electrograms and atrial fibrosis in persistent atrial fibrillation: Combined magnetic resonance imaging and high-density mapping. J Am Coll Cardiol. 2013;62(9):802-812. doi:10.1016/j.jacc.2013.03.081

23. NDREPEPA G, SCHNEIDER MAE, KARCH MR, et al. Impact of Atrial Fibrillation on the Voltage of Bipolar Signals Acquired from the Left and Right Atria. Pacing Clin Electrophysiol. 2003;26(4p1):862869. doi:10.1046/j.1460-9592.2003.t01-1-00151.x

24. Rodriguez-Manero M, Valderrabano M, Baluja A, et al. Validating Left Atrial Low Voltage Areas During Atrial Fibrillation and Atrial Flutter Using Multielectrode Automated Electroanatomic Mapping. JACC Clin Electrophysiol. 2018;4(12):1541-1552. doi:10.1016/j.jacep.2018.08.015

\section{TABLES}

Table 1. Clinical characteristics

\section{Clinical characteristics $(\mathrm{N}=\mathbf{2 3})$}

\begin{tabular}{ll}
\hline Age $($ mean $\pm \mathrm{SD})$ & $59,2 \pm 7,6$ years \\
Sex (male) & $74 \%$ \\
AF time of evolution $($ mean $\pm \mathrm{SD})$ & $4,5 \pm 3,8$ years \\
HTA & $52 \%$ \\
DM & $9 \%$ \\
Dyslipidemia & $39 \%$ \\
Smoking & $39 \%$ \\
Sleep apnoea syndrome & $13 \%$ \\
Left atrium enlargement & $87 \%$ \\
\hline
\end{tabular}

\section{FIGURE LEGENDS}

Figure 1: LVA extension $<0.5 \mathrm{mV}$ : comparison between maps in $\mathrm{AF}$ and maps in SR.

Figure 2: LVA extension $<0.3 \mathrm{mV}$ : comparison between maps in $\mathrm{AF}$ and maps in $\mathrm{SR}$.

Figure 3A: Comparison between $\mathrm{AF}$ and $\mathrm{SR}$ maps for the $0.3 \mathrm{mV}$ threshold. Postero-anterior projection. Patient 1.

Figure 3B: Comparison between $\mathrm{AF}$ and $\mathrm{SR}$ maps for the $0.5 \mathrm{mV}$ threshold. Postero-anterior projection. Patient 1.

In both images, we observed an appropiate match in LVA location, although LVA extension is greater in the AF map.

Figure 4A: Comparison between AF and SR maps for the $0.3 \mathrm{mV}$ threshold. Superior projection. Patient 2.

Figure 4B: Comparison between AF and SR maps for the $0.5 \mathrm{mV}$ threshold. Superior projection. Patient 2.

In both images, we observed lack of LVA in SR maps, for $0.3 \mathrm{mV}$ threshold as well as for the $0.5 \mathrm{mV}$ threshold. In this case it is not possible to assess if LVA location matches appropriately.

Figure 5: Kaplan-Meier arrhythmia free survival. LVA indicates low voltage areas. 
LVA $<0.5 \mathrm{mV}$ COMPARISON

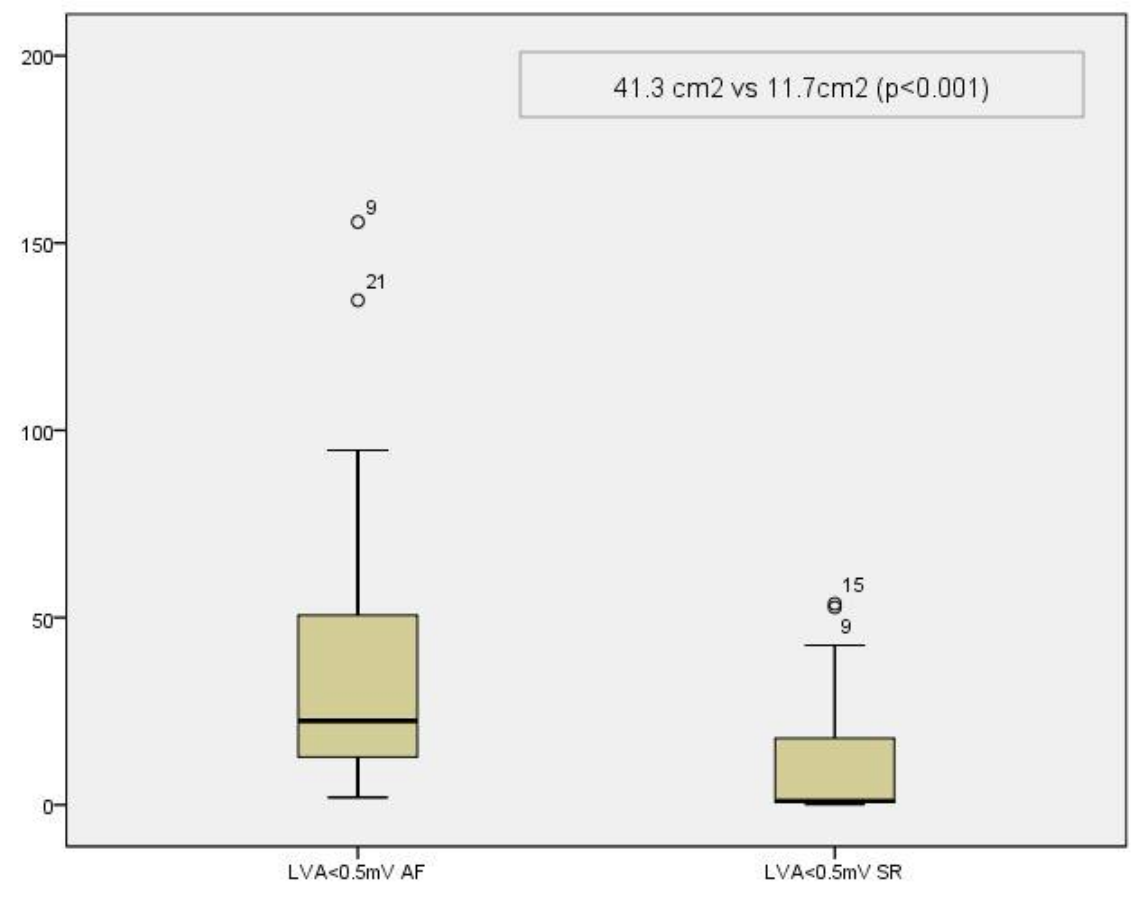

LVA $<0.3$ mV COMPARISON

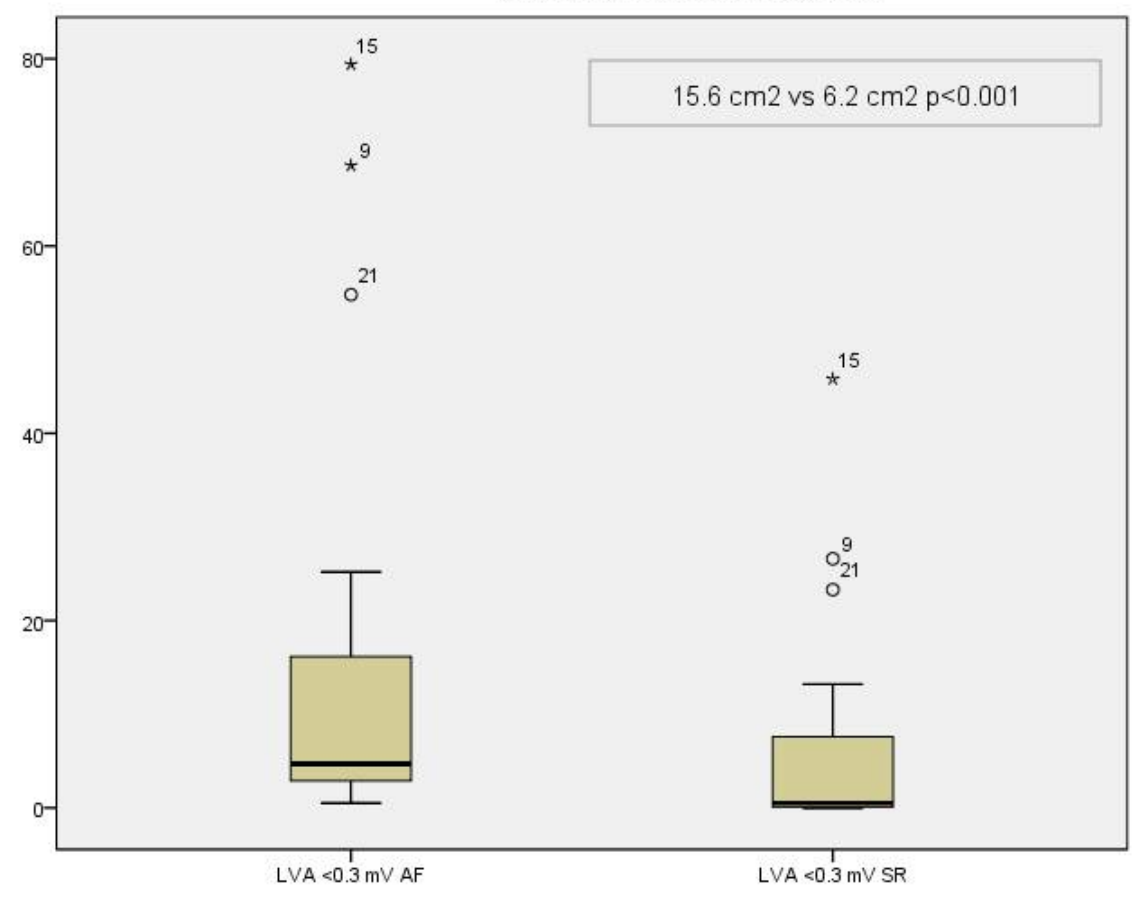




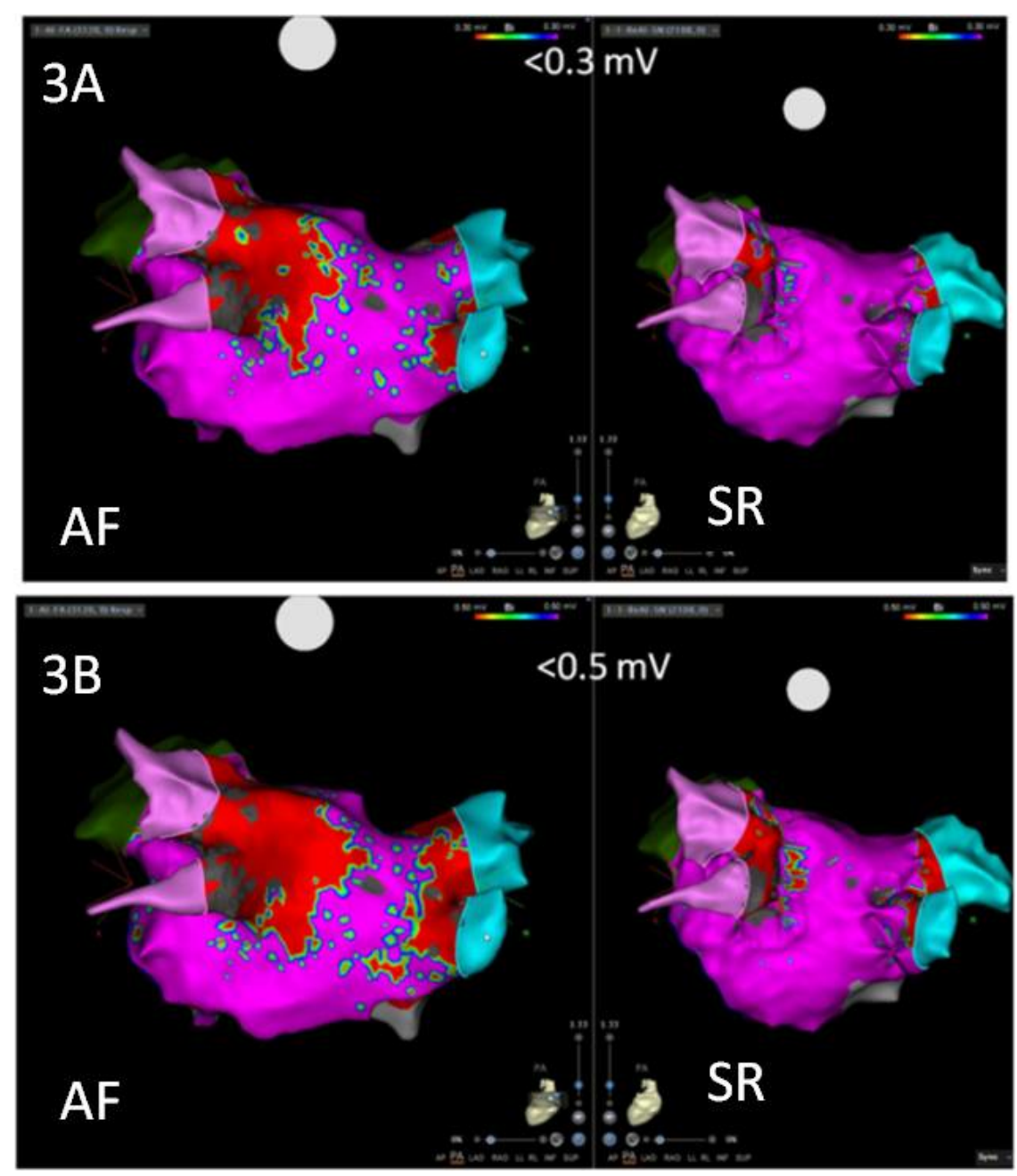



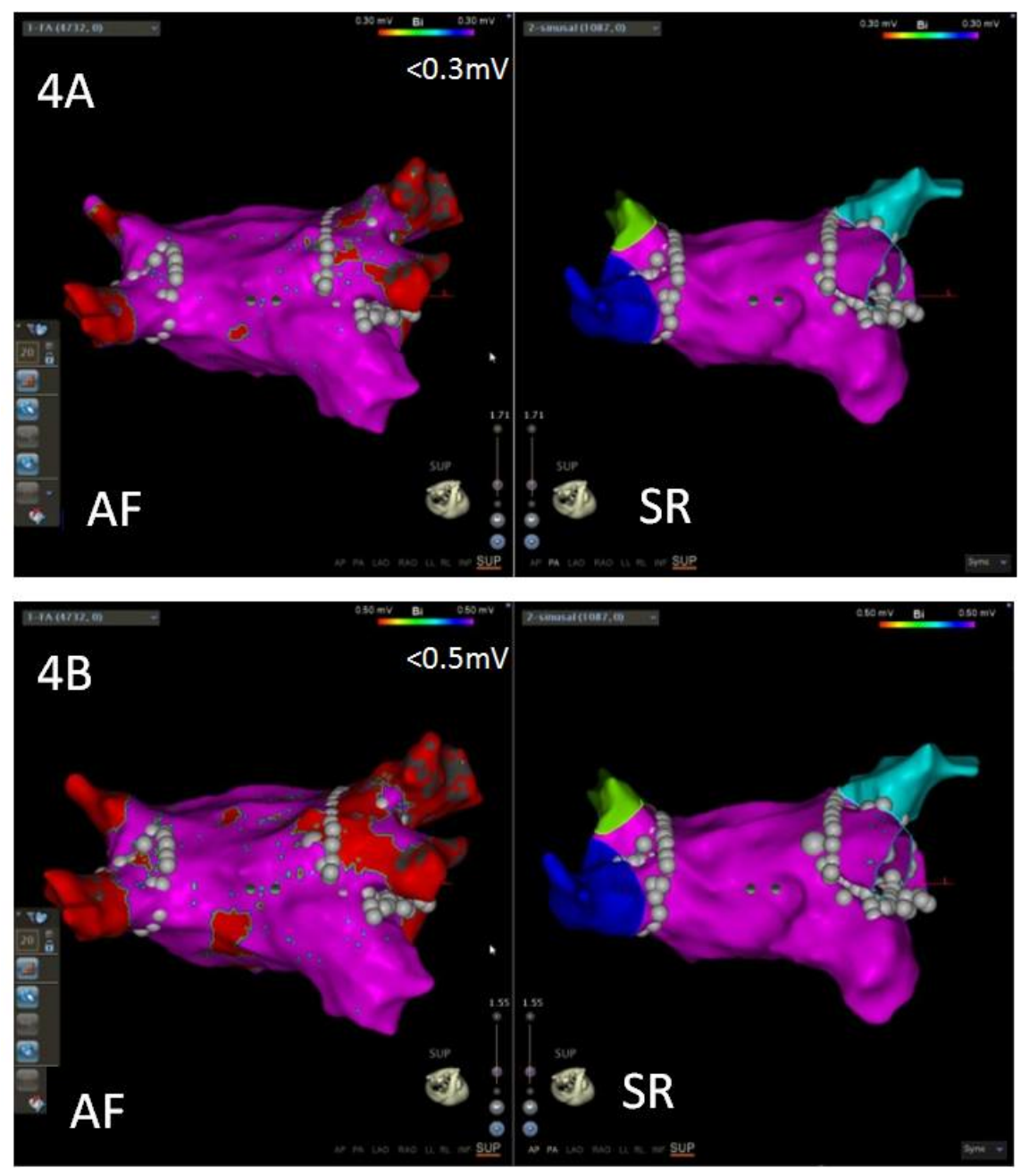


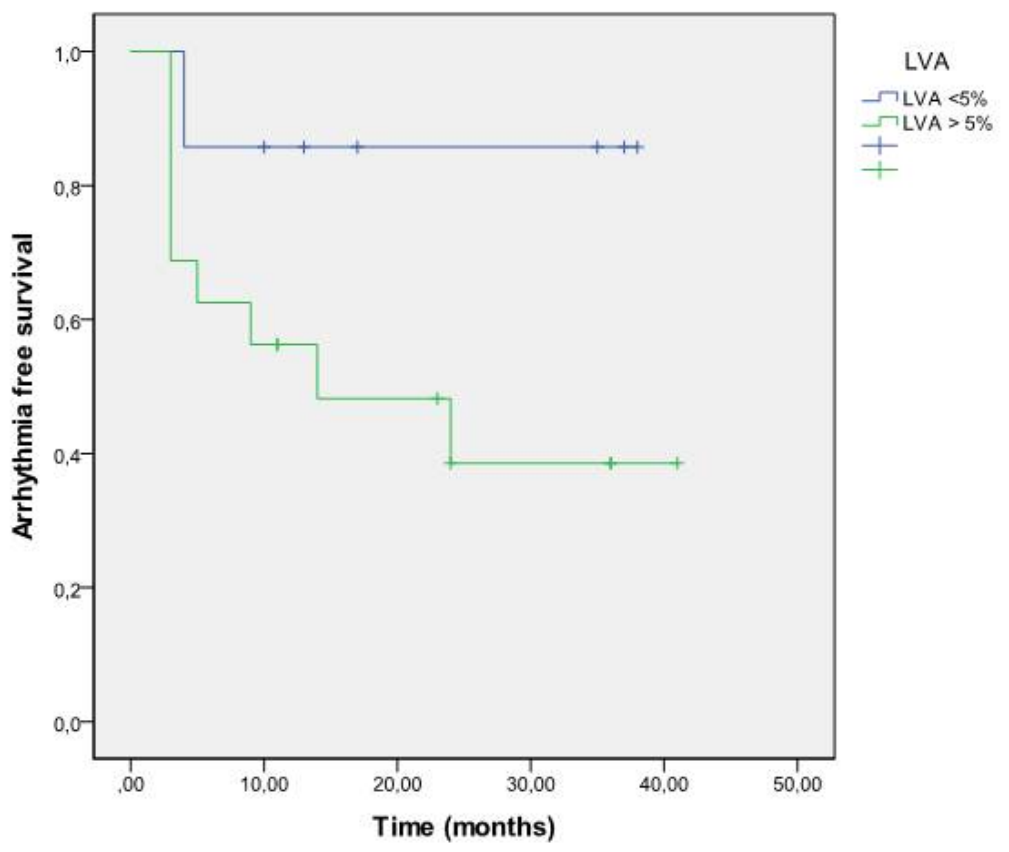

